\title{
Les territorialisations du dancehall jamaïcain.
}

\section{Romain Cruse}

\section{(QpenEdition \\ Journals}

\section{Édition électronique}

URL : http://journals.openedition.org/echogeo/11737

DOI : $10.4000 /$ echogeo. 11737

ISSN : 1963-1197

\section{Éditeur}

Pôle de recherche pour l'organisation et la diffusion de l'information géographique (CNRS UMR 8586)

\section{Référence électronique}

Romain Cruse, "Les territorialisations du dancehall jamaïcain. », EchoGéo [En ligne], Sur le Vif, mis en ligne le 03 mars 2010, consulté le 20 avril 2019. URL : http://journals.openedition.org/echogeo/11737 ; DOl : 10.4000/echogeo. 11737

Ce document a été généré automatiquement le 20 avril 2019.

\section{(c) (i) (9)}

EchoGéo est mis à disposition selon les termes de la licence Creative Commons Attribution - Pas d'Utilisation Commerciale - Pas de Modification 4.0 International 


\title{
Les territorialisations du dancehall jamaïcain.
}

\author{
Romain Cruse
}

\section{NOTE DE L'AUTEUR}

L'auteur remercie le photographe Romain Philippon pour lui avoir permis d'utiliser certains de ses clichés (http://www.pendantcetemps.fr/).

C'est par le bruit que la Caraïbe est entrée en moi. J'avais oublié ce vacarme. Cette foule hurlante. Ce trop plein d'énergie. Ville de gueux et de riches.

Debout avant l'aube...

Si on meurt plus vite qu'ailleurs la vie est ici plus intense. Chacun porte en soi la même somme d'énergie à dépenser sauf que la flamme est plus vive quand son temps pour brûler est plus bref.

Dany Laferrière, L'énigme du retour.

1 L'espace du dancehall jamaïcain est profondément multidimensionel. Ceci tient d'abord à la polysémie du terme : le dancehall désigne en effet à la fois l'espace physique où se tiennent les «danses »- littéralement le hall de danse -, mais c'est aussi l'espace sonore qui occupe cet espace physique, qui l'emplit et qui lui donne corps, ainsi que dans un sens plus strict une étape de l'évolution de la musique jamaïcaine émergeant dans les années 1980 (le genre dancehall) (Stolzoff, 2000 ; Katz, 2003 ; Lesser, 2008). C'est à la fois un contour délimité par des marqueurs géographiques (barrières, guichets, sécurité, etc.) et culturels (isoglosse ${ }^{1}$, etc.) et son contenant sonore (la musique dancehall). Il désigne donc aussi bien le contenant que le contenu.

2 La multidimensionalité du dancehall jamaïcain est aussi due à sa présence et à son influence dans de nombreuses sphères de la société jamaïcaine. Économiquement, le 
dancehall est là encore un espace à part. La plupart des artistes, la multitude de petits vendeurs qui entourent l'événement, les danseuses, les techniciens et autres, qui animent cette petite économie de survie la rattachent au vaste espace de l'économie informelle. L'explosion internationale du reggae derrière Robert Nesta «Bob» Marley, puis des formes contemporaines de musique jamaïcaines (le dancehall au sens strict) à la suite des premiers artistes jamaïcains décorés de grammies aux États-Unis (Shaba Ranks 1991 « Raw as ever ", etc.) ont entrainé une reprise en main des échelons les plus rentables de l'« industrie musicale » (production, management, etc.) par l'élite économique jamaïcaine et son glissement vers la sphère légale et formelle. Cependant, pour la vaste majorité de ses acteurs - y compris la très grande majorité des chanteurs et musiciens -, l'espace économique du dancehall demeure un espace informel.

Le dancehall est aussi l'espace d'une contre-culture. Cette contre-culture est paradoxalement la culture d'une « minorité majoritaire », celle des classes noires ( $91 \%$ de la population) et pauvres (au moins $3 / 4$ de la population, avec des variations selon les critères retenus). Cette culture remet en cause la « jamaïcanité » telle que les élites claires ont tenté de l'imposer depuis plusieurs siècles. Au centre de cette remise en cause s'impose le langage même des jamaïcains, localement connu sous l'étiquette péjorative de «patwa » - qui est incontestablement, avec le dancehall, le langage premier des jamaïcains. Ce langage n'a réellement sa place ni à l'école - bien que de nombreux professeurs soient bien incapables d'apprendre à leurs élèves l'«anglais de la reine» imposé par les programmes scolaires -, ni au travail, ni dans l'administration. Dans la rue, chez les particuliers et dans l'espace du dancehall il demeure le langage officiel. Un isoglosse entoure donc le dancehall, délimitant une frontière linguistique et identitaire marquant le dernier bastion, jusqu'ici imprenable, de la culture jamaïcaine et de sa résistance. Un exemple souligne l'importance culturelle de cet isoglosse : le « je » jamaïcain. En effet si pour les autres pronoms le système anglais a été suivi de plus ou moins près (on retrouve notamment le « you » singulier, le « him » en lieu et place du he/she/it, le we, etc.), le « je » jamaïcain (« mi ») - qui est aussi le « je » des créoles francophones, est africain. C'est un dérivé du langage twi du Ghana contemporain (Cassidi, 1971). Symboliquement, le « patwa » affirme donc l'africanité du sujet qui s'exprime.

Politiquement enfin, le dancehall est le lieu où s'informent ces classes pauvres, le lieu où n'a pas siège l'autorité de l'État et son pouvoir de propagande (n'avait pas du moins). Bob Marley disait par exemple du reggae qu'il est « le journal des pauvres ». Norman Stolzoff (2000) voit même dans ces rassemblements une sorte d'Église, ou plus exactement de contre-Église si l'on suit son raisonnement. Il existe une longue tradition de titres remettant en cause la version étatique de l'histoire globale et de l'histoire locale, ainsi que des violents affrontements politiques locaux (Cruse, 2009). Les milieux politiques jamaïcains ont cependant bien compris l'importance stratégique de cet espace tout comme les Duvalier connaissaient son importance en Haïti - ils ont laissé derrière eux l'héritage de la politique du koudyay, une politique culturelle axée sur la musique, à mi-chemin entre propagande et corruption (Averill, 1997). On retrouve des développements similaires avec R. Trujillo en République Dominicaine (idem), B. Panday à Trinidad (Cruse, 2009)... L'espace multidimensionel du dancehall (aussi bien l'espace physique que l'espace sonore) est donc, on le voit et on le verra plus en détail, l'objet d'enjeux et de rivalités de territorialisation. 


\section{Origines et évolutions du dancehall jamaïcain}

5 On doit à Norman Stolzoff et à Beth Lesser les analyses les plus précises et détaillées du dancehall Jamaïcain. Beth Lesser analyse le style musical Dancehall au sens strict, comme celui qui explose à Kingston dans les années 1980 et conquiert par la suite les Etats Unis et la Grande Bretagne, vectorisé par l'émigration jamaïcaine, particulièrement celle des gangs. Lesser retrace ses origines jusqu'aux années 1950 (Lesser, 2008). N. Stolzoff (2000) va plus loin dans l'analyse. Il est le premier à avoir introduit la notion de «dancehall » en tant que genre musical présent en Jamaïque depuis la période esclavagiste, en réajustement constant sous les apports - musicaux, culturels et techniques - étrangers. Stolzoff qui appuie son essai sur un très long et très minutieux travail de terrain auprès des principaux acteurs (soundmen, Deejays, danseuses et autres acteurs de l'économie informelle gravitant autour de l'activité, etc.) et de nombreuses interviews de vétérans de la musique jamaïcaine, emploie donc le terme de dancehall comme synonyme de «musique jamaïcaine». Le lieu particulier d'échange, l'«arène» dans laquelle se déroulent les multiples «clash»- les affrontements qui caractérisent le dancehall -, ressort de son étude comme l'élément primordial et le fil d'Ariane de la musique jamaïcaine au point que l'on peut effectivement qualifier celle-ci, de manière générique, comme le dancehall. L'espace physique, la clôture spatiale, donne donc son nom au genre musical dans son ensemble.

\section{Créolisation ${ }^{2}$ et évolution de la musique jamaïcaine}

6 Si on accepte cette définition de la musique dancehall comme l'évolution permanente de la musique des classes pauvres jamaïcaines, comme le propose Norman Stolzoff , on retient donc un fait important. Le dancehall, en tant que musique, naît en Jamaïque durant la période esclavagiste. Ses premières formes sont des imitations de la musique des planteurs (les origines de la " mimicry»), musique que certains esclaves sont entrainés à reproduire durant les réceptions. Il existe un débat plus large dans la Caraïbe qui oppose des universitaires comme le trinidadien V.S. Naipaul (prix Nobel de littérature en 2001), reproduisant l'avis régnant du temps de la colonie, et selon lequel les classes pauvres antillaises sont caractérisées par leur piètre et affligeante tentative d'imitation des maitres - le mythe du « caribbean mimic man »-, et des auteurs comme le St. Lucien Dereck Walcott (prix Nobel de littérature en 1992 et professeur de poésie à Harvard), qui ont souligné le caractère ironique de ces imitations. En effet, dès les balbutiements du dancehall jamaïcain, si les esclaves imitent la musique de leurs maîtres c'est souvent pour mieux les railler. En réalité, les esclaves préfèrent des styles musicaux métissés, liant ces formes à leur culture musicale faite de percussions, et respectant l'importance des contretemps (inconnus de la musique européenne). Par comparaison avec la création des formes linguistiques Antillaises, ce métissage a été décrit avec justesse comme une «créolisation musicale». Comme le rappelle Gage Averill (1997) dans son magnifique ouvrage sur les rapports entre la musique et le pouvoir haïtien, cette créolisation musicale a crée dans chaque île, encore une fois comme dans le domaine linguistique, une forme d'expression propre mêlant de façon originale ses différents héritages pour donner naissance à un style propre : le Calypso et le Soca à Trinidad, le Kadans-lypso à la Dominique, le Zouk dans les Antilles francophones, la Rumba, le Bolero ou encore le Son à 
Cuba, le Merengue en République Dominicaine, le Rara, la Misik Rasin ou la Kadans et le Kompa en Haïti...

7 La créolisation linguistique jamaïcaine a donné naissance au (mal-nommé) «patwa » (le terme lui même originaire du français et écrit selon l'orthographe du créole francophone, souligne la créolisation), « créole afro-jamaïcain » semblant être un meilleur compromis dénominatif (Adams, A., 1991). Sa créolisation musicale a engendré le dancehall. Le dancehall n'a cependant pas toujours été connu sous le nom de la piste sur laquelle il se pratique, se produit et se consomme. Nous l'avons dit, ses premières formes sont un dérivé de musiques et de danses européennes. Le premier style de créolisation musicale à émerger est le Mento : c'est la sixième forme de Quadrille, danse de cour française originaire des campagnes anglaises. Plus qu'une danse, c'est une contredanse, qui emprunte aux colons européens un air et quelques instruments, que les esclaves greffent sur leurs rythmiques propres, jouées avec les instruments dérivés de leur culture musicale (rumba box, tambour, etc.). Dans les dancehalls du Mento, on raconte les histoires de la vie courante, des histoires d'amour, des histoires pour se donner du courage, et des histoires pour se moquer de la vie sur la plantation. Pour échapper à la répression des planteurs, le langage utilisé par ces griots déplacés est imagé, et lui aussi subit un processus de créolisation créatif. C'est à ce point de rencontre, dirait l'écrivain guyanien Wilson Harris (1970), que l'on prend conscience de la proximité entre les « arts de la coexistence créatifs » créoles qui ont donné naissance en même temps, et suivant la même dynamique, à une musique imagée et à une langue poétique.

8 Les maîtres ne sont pas dupes, eux aussi apprennent ces langages créoles pour contrôler leurs esclaves et ils ont leurs indicateurs au sein de la population servile. Mais ces espaces de liberté, aussi restreints soient-ils, agissent comme une soupape de sécurité indispensable. Un peuple qui rit et qui chante ne conspire pas pense-t-on alors chez les planteurs. Un tiers des rebellions sur les plantations britanniques sont pourtant fomentées dans les dancehalls, particulièrement durant les fêtes de Noël...

9 Puis s'ajoutent des apports musicaux nouveaux. À partir du début du $\mathrm{xx}^{\mathrm{e}}$ siècle des travailleurs jamaïcains migrent vers les États-Unis, d'autres sont embauchés à la construction du canal de Panama, des coupeurs de canne sont envoyés à Cuba, des militaires partent en Grande Bretagne pour soutenir l'effort de guerre, etc. De ces voyages les jamaïcains ramènent de la musique, particulièrement du jazz américain, de la rumba cubaine, un peu de rock'n roll. Plus que des disques ils ramènent ce que les musiciens nomment un "feeling », ainsi qu'un goût prononcé pour les musiques créatives - déjà des formes créoles - de la diaspora afro-américaine.

10 L'évolution ne s'est pas arrêtée au reggae des années 1970, bien que sa popularité croissante ait entraîné sa perpétuation en parallèle des nouveaux styles développés depuis, principalement influencés par le Hip Hop étasunien (bien que dans ce processus l'influence soit notable dans les deux sens, les posses des années 1990 ayant laissés leur empreinte aussi dans l'espace culturel noir américain). La multiplication des revenus générés par la musique en Jamaïque, et la baisse du coût des ordinateurs, puis le développement des logiciels d'enregistrements et de création artistique ont entrainé la création d'un style de musique qui s'émancipe des instruments traditionnels. Place tout d'abord à des rythmiques reggae "digitales » (début des 1980's), puis rapidement à des versions de plus en plus minimalistes et à une profusion de chanteurs, deejays et autres singjays. La musique contemporaine jamaïcaine naît dans les années 1980 et prend alors le nom de son espace de prédilection : le dancehall. 


\section{Evolution du lieu du dancehall}

11 de la plantation - à caractère profondément rural donc -, il s'est déplacé vers les centres urbains, plus particulièrement vers certains espaces des centres urbains. Une constante, l'espace du dancehall est un espace de l'exclusion. À Kingston par exemple, il est interdit de tenir une "session" au dessus de la ligne (imaginaire) crossroad - halfwaytree, ligne connue de tous les jamaïcains comme la frontière tacite entre la ville haute, riche et relativement calme (uptown), et la ville basse, pauvre et sulfureuse (downtown) (Cruse, 2009). Comme dans les plantations de l'époque coloniale, il s'agit donc en réalité d'une dérogation : « un esclave qui s'amuse est un esclave qui ne se rebelle pas » disait le gouverneur espagnol de Porto Rico. est une ruelle (Lane) qui fait à la fois fonction d'impasse vis à vis du trafic automobile - le mode de déplacement des riches jamaïcains et des forces de l'« ordre » (le guillemet rappelle que les forces armées jamaïcaines sont responsables d'un meurtre perpétré dans l'île sur cinq d'après les statistiques officielles !) -, et de carrefour pour la multitude d'acteurs de l'économie informelle et illégale qui animent cet espace. Comme dans une forêt balisée d'une ramification de pistes et de sentiers invisibles ou presque à l'œil étranger, ces espaces en apparence clos se révèlent en effet la base des réseaux de chemins et d'échappatoires invisibles au premier abord. Les frontières des uns sont les réseaux de communication des autres. Ici la plaque de zinc d'une clôture n'est pas fixée et ouvre sur une arrière-cour, qui ouvre elle même sur une ruelle voisine, là une parcelle en friche ouvre l'accès d'un quartier voisin, ailleurs en ouvrant la porte d'une arrière-cour on accède directement à la montagne, à la mangrove, à un canal d'évacuation des eaux usées, etc. Ainsi, lorsque les forces de police, les squadies ou l'armée - autres éléments incontournable de toute session -, s'introduisent par l'entrée de la ruelle et mettent en place barrage et fouille, vendeurs de ganja, gunmen et autres « hustlers » s'échappent à travers les pores de cet espace aux bordures à perméabilité variable.

13 La route principale, qui relie le quartier au reste de la capitale, et sur laquelle circulent bus et taxis collectifs, n'est jamais très loin, sans quoi la session peine à attirer la foule compacte qui caractérise cet espace à partir du milieu de la nuit et jusqu'aux premiers rayons de soleil. Un tag sur un mur de l'artère principale indique généralement la tenue de ces sessions de ruelle aux noms évocateurs "Weddy Wednesday ", " Daggerin' tuesday », " Passa Passa », etc.

Le lieu de référence du dancehall demeure donc antinomique du lieu de référence de l'élite jamaïcaine. Celle ci n'échappe cependant pas à la propagation des lourdes basses propagées par les systèmes de sonorisation surpuissants des sound system - jusqu'à 10000 volts pour les plus puissants. Le dancehall se tient pourtant à l'extrême opposé de l'échelle socio-spatiale, au fond de la ruelle des quartiers périphériques. Périphérique est ici une image, puisque les ghettos de Kingston, qui regroupent $2 / 3$ de la population de la capitale jamaïcaine, sont avant tout centraux (Cruse R., 2009). Ils occupent tout le centre ville ainsi que les artères qui partent de ce centre vers Spanish Town à l'Ouest et Bull Bay à l'Est, tous les espaces délaissés par les promoteurs immobiliers et les riches propriétaires en raison de la chaleur, des inondations, et de l'insalubrité générale. Du lotissement en ruines (Tivoli Gardens) au véritable bidonville élevé sur une décharge publique (Riverton)

EchoGéo, Sur le Vif | 2010 
ou sur la mangrove (Seaview Gardens), en passant pas les anciens beaux quartiers fuis par leurs riches habitants en raison de la violence politique (Rockfort), tous ces espaces sont appelés en Jamaïque "ghettos", ou "garnisons", en référence à l'unicité ethnique, économique et au garnisonnement politique typique.

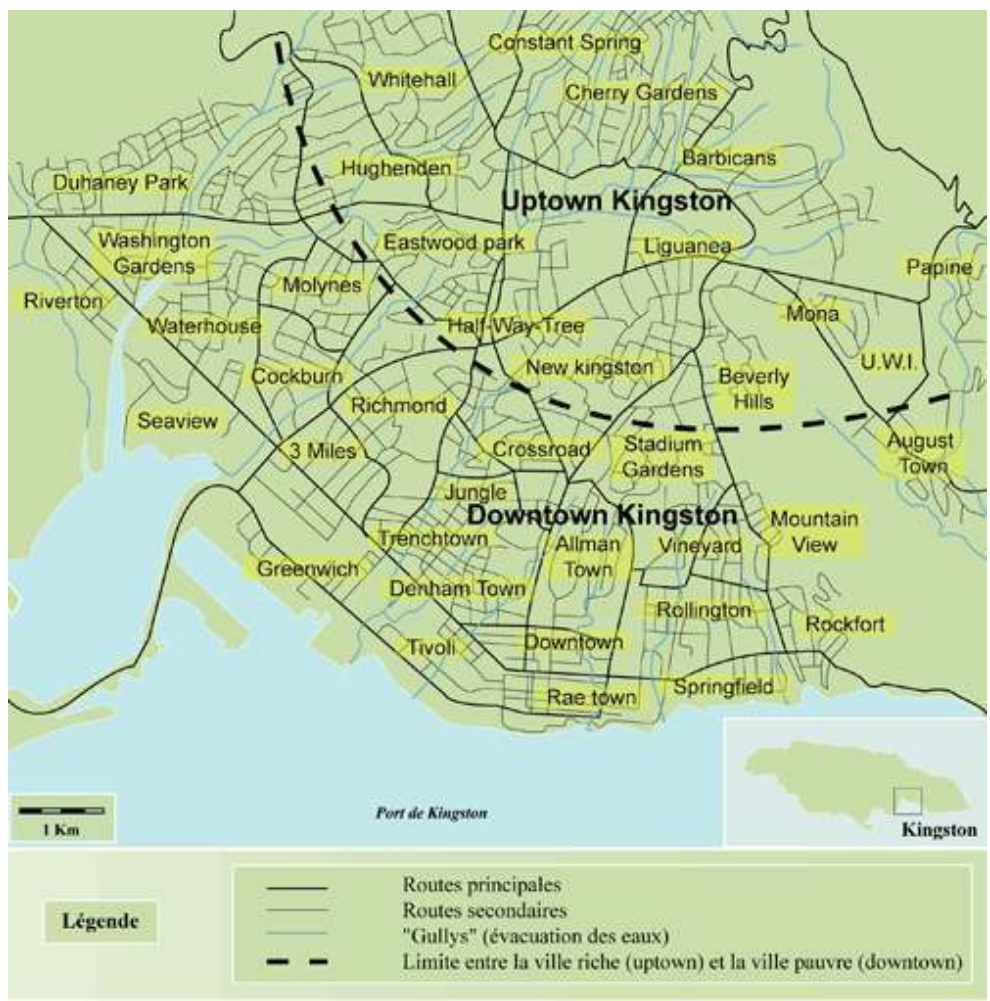

Source : Romain Cruse

Image2À la campagne, la logique spatiale est inverse puisqu'il faut avant tout réussir à attirer une foule. Le dancehall occupe alors des espaces centraux tels que des terrains de football situés à proximité directe des principaux carrefours. Toutefois, on privilégie là encore des espaces aux bordures poreuses...

L'évolution de l'espace du dancehall, d'un abattis abrité derrière la plantation au cœur des ghettos urbains, est évidemment lié à l'évolution démographique de l'île dans laquelle Kingston a fini par absorber plus ou moins deux tiers des habitants. Ce gonflement prodigieux eut lieu à l'époque où les espaces de migrations traditionnels, tels que les É tats-Unis ou la Grande Bretagne (mais aussi Panama, Cuba, etc.), arrêtèrent, pour différentes raisons, d'absorber plus encore l'exode rural massif des Jamaïcains. Elle est aussi liée à un changement dans la forme même du dancehall. Autrefois pratiqué par un groupe d'instrumentistes, la musique jamaïcaine a en effet connu une véritable révolution à la fin de la Seconde Guerre mondiale. C'est alors que, de manière simultanées, des anciens techniciens de l'armée britannique rentrèrent chez eux avec leur nouveau bagage technologique, que de nombreux musiciens jamaïcains seront pris dans le flot de travailleurs embauchés pour la reconstruction des villes bombardées en Grande Bretagne, et que des migrants commencèrent à ramener en Jamaïque de la musique afro-américaine sous forme de disques vinyles. Par ailleurs, en centre ville de Kingston, les commerçants au détail - qui vendent tous plus ou moins les mêmes rares et chers produits importés- se livrent une véritable bataille rangée pour attirer les foules dans leur magasin. Ils remarquent qu'un poste de radio attire invariablement un 
attroupement devant leur commerce. Pour attirer ces foules, il faut donc diffuser de la musique, jouer les meilleurs morceaux, plus fort, et avec une meilleure qualité sonore... C'est ainsi que naissent les premiers sound systems, propriétés de commerçants chinois qui embauchent les techniciens formés dans l'armée britannique pour leur monter des systèmes de sonorisation (sound system) performants, et importent des disques de souls et de jazz d'Amérique du Nord. Face à l'engouement du phénomène, ces commerçants détachent rapidement ces sound system de leurs façades pour organiser les premières véritables sessions dans des espaces vacants environnants. La demande de ces sound system en morceaux inédits et originaux crée un véritable foisonnement artistique dans l'île et un renouveau de la pratique musicale. Métissant les genres cubains (boléro, son, etc.), jamaïcains (mento, etc.), et nord américains (jazz, soul), les musiciens jamaïcains créent une succession de styles de dancehall depuis le ska, le rock steady, pour finalement aboutir au « reggay » - qui deviendra reggae - à la fin des années 1960.

L'évolution des lieux du dancehall nous amène donc à sa marchandisation, avec l'apparition des premiers sound-system au début du $\mathrm{xx}^{\mathrm{e}}$ siècle. D'une contre culture, on bascule alors dans une contre-économie.

\section{De la contre-culture à la contre-économie}

La base d'une société de type plantation, la "plantalogique », repose sur la séparation entre deux mondes distincts, bien que connectés. Le premier est celui des planteurs, puis bientôt aussi de certains enfants nés des relations plus ou moins cachées, plus ou moins contraintes, et plus ou moins émancipatrices, entre planteurs et femmes esclaves : les mulâtres. Ce monde vit enfermé entre un sentiment de supériorité raciste et la peur latente d'un soulèvement des esclaves, forts et nombreux. Il fonctionne bien souvent selon le principe de Willie Lynch, un ancien planteur jamaïcain qui conseillait à ses semblables d'attribuer à ses travailleurs un gradient de traitement relatif au degré de pigmentation pour diviser la classe noire. Celle-ci représente l'autre monde, celui des résistants et résignés. Constante caribéenne, cette division sociale, économique, ethnique et spatiale s'est maintenue à travers le processus politique d'indépendance en raison de l'hermétisme de la caste « blanche » et aisée et des héritages politiques. On retrouve par exemple ces descendants de colons du $\mathrm{xvIII}^{\mathrm{e}}$ siècle, " en race pure» selon l'ignoble expression du béké ${ }^{3}$ Alain Huyghues-Despointes ${ }^{4}$, aussi bien à Cap-est (Martinique) qu'à Carenage (Trinidad) ou Beverly Hills (Jamaïque). Cette petite minorité a partout cherché à imposer sa propre culture, elle-même inévitablement métissée, comme la base culturelle de ces espaces. En Jamaïque comme en Haïti par exemple, cette élite a longtemps tenter d'imposer la langue coloniale comme langue officielle, rejetant les langages créoles locaux comme des symptômes de la "mimicry " (angliscisme qu'on peut traduire par piètre imitation, ou "singerie »), témoignant de l'incapacité - sans doute génétique - à intégrer les bases de la "civilisation». Comme le "patwa», le dancehall jamaïcain continue ainsi d'être dénigré par ces milieux qui ont voulu imposer la jamaïcanité comme une « afrosaxonité » tropicale.

Ce rejet n'a rien d'étonnant puisque le dancehall est avant tout l'espace de la contreculture, la culture de la " minorité-majoritaire ». Le poids des classes pauvres comparé à l'isolement des quelques familles de descendants de colons - une vingtaine de familles " claires » contrôle au moins $2 / 3$ des entreprises jamaïcaines -, fait de cette espace le lieu officiel d'expression du créole jamaïcain, riche langage imagé ayant conservé formes et 
expressions africaines auquel ont été intégrées les bases logiques de la conjugaison britannique (les verbes à la forme simple, les auxiliaires pour former les temps, mais pas les exceptions par exemples), ainsi que de rares héritages francophones probablement liés à la fuite des colons de Haïti durant la première « révolution noire " (Farmer, 2006). Tandis que les classes aisées se délectaient du rock'n roll américain, les sound system diffusaient la musique jamaïcaines dans les dancehalls, et les goûts musicaux évoluèrent différemment jusqu'à ce que le dancehall contemporain (le sens strict rejoint ici l'appellation générique), dans ses formes les plus crues et ouvertement sexuelles, ne conquièrent une partie du marché américain. Par ricochet, la fièvre du dancehall se répandit dans les quartiers riches qui ne pouvaient de toutes façons plus se protéger des débordements sonores et de l'invasion des chaînes de radio et des canaux hertziens par le phénomène.

L'extension du dancehall aux marchés riches, locaux et étrangers, entraîna une croissance fulgurante des profits tirés de la musique jamaïcaine. C'est à partir des années 1990 qu'on commença en Jamaïque à parler d'« industrie musicale », alors que les gangs de la drogue « jaméricains » perfusaient d'un coté les réseaux de production, en Jamaïque, et aidaient à la diffusion aux États-Unis. Alors que l'aspect contre-culturel s'estompait petit à petit avec la contagion du "slack » (littéralement "relâchement ", caractérisé par des paroles très ouvertement sexuelles) promu par certaines élites politiques et des "gun songs", l'aspect contre-économique prit le devant. L'espace du dancehall devint rapidement prédominant pour une foule d'employés déçus par la misère générée par les emplois dans les zones franches industrielles de la capitale et dans les enclaves touristiques de la côte Nord. L'énorme marché du dancehall crée une demande gigantesque allant de la simple fourniture de boisson et de nourriture autour des événements, au design des tenues des danseuses, à la formation des musiciens et techniciens en passant par la disponibilité des vastes scènes pour les talents locaux, de potentialités énormes pour les studios d'enregistrements, les producteurs, etc.

Rapidement cet espace fut scindé en deux parties distinctes et quasi hermétiques. D'un coté les parties lucratives comme l'enregistrement, le management et la promotion furent affectées à l'économie légale, taxées et protégées, et largement investies par les classes aisées. De l'autre, toutes les activités peu lucratives furent abandonnées à l'économie informelle qui nourrit en Jamaïque plus de la moitié de la population (et contribue à $43 \%$ du PNB) selon les estimations basses de la Banque de Développement Interaméricaine.

\section{Géopolitique du dancehall}

Les enjeux géopolitiques - les rivalités dont l'enjeu est l'espace - qui animent l'espace du dancehall peuvent être analysés le long de deux axes : leur envergure spatiale et leur temporalité. Le dancehall est en effet l'espace de différentes luttes métaphorisées qui prennent place à différentes échelles (ex. : l'opposition de deux artistes à l'échelle la plus grande, l'opposition idéologique entre "capitalistes » et "socialistes", dans les années 1970, à l'échelle la plus petite), d'où, vraisemblablement, sa propension aux analogies meurtrières (généralement en lien avec le feu et les armes). Ces luttes peuvent donc se classer sur un premier axe par échelle, par ordre décroissant : clash entre artistes / danseuses, etc. (Stolzoff, N., 2000) ; conflit de classes sociales entre et participants et détracteurs (idem), batailles pour l'affirmation des genres (définitions de la masculinité et 
de la féminité disputés dans les dancehalls entre hommes et femmes) (Cooper, C., 1994 ; 2000) ; lutte idéologique (Cruse, R., 2009), etc.

Cet axe spatial est doublé de l'axe qui lui est inséparable, celui du temps qui passe. Car ces luttes, quelles que soient leurs échelles, évoluent. C'est cet axe qui nous guidera dans cette partie géopolitique, particulièrement la période charnière des années 1970/1980 (glissement de la proéminence du « reggae conscient » vers le dancehall orienté sur le clash) qui permet d'expliciter les développement de la période actuelle.

\section{De l'affrontement idéologique des années 1970 au clash entre reggae conscient et dancehall slack}

23 Le premier ministre socialiste Michael Manley décrivait le reggae comme «le langage premier des jamaïcains». Son parti ne manqua d'ailleurs pas d'instrumentaliser - si l'on peut dire - cette musique à des fins électoralistes, sans jamais pour autant atteindre les sommets du parti conservateur, le JLP, dont les hauts dirigeants possédaient, et possèdent toujours, certains des principaux studios d'enregistrements de l'île (Cruse, 2009). C'est au parti libéral qu'on doit en effet la promotion, locale et internationale, des dancehalls " slacks ", aux allusions ouvertement sexuelles, qui possèdent le double avantage de garder dans l'ombre le message révolutionnaire du reggae des années 1970 et de promouvoir dans l'île le tourisme, notamment sexuel (O'Connell, J., Taylor, J., 1999). A ce croisement temporel se recoupent les deux échelles géopolitiques du genre et de l'idéologie politique évoqués en introduction. Dans les années 1980, un village de pêcheur situé à quelques dizaines de kilomètres au Sud-ouest de Montego Bay sera profondément bouleversé par cette évolution. Négril deviendra ainsi en moins de dix ans, d'après les spécialistes entourant la chercheuse K. Kempado, une destination incontournable du tourisme sexuel masculin, puis féminin (phénomène célébré par l'expression "Rent-a-dread») (Mullings, 1999 ; O'Connell, Taylor, 1999). Sa longue plage de sable blanc est colonisée par les hôtels étrangers et « all inclusive » et une " police touristique » patrouille pour s'assurer du bon déroulement de la ségrégation. Si la musique jamaïcaine a toujours donné une place au « relâchement » (slack), son instrumentalisation par l'administration conservatrice lui a donné une nouvelle résonance.

24 L'ancien premier ministre conservateur E. Seaga fut aussi longtemps une figure incontournable du dancehall jamaïcain (Lee, 1999 ; 2004). Ce fils de migrant libanais né né à Boston (mais baptisé en Jamaïque), profita de ses études à Harvard - il se spécialise en anthropologie dans la culture traditionnelle jamaïcaine - pour faire tout d'abord commerce de disques rares de musique afro-américaine qu'il revend aux premiers sound systems lorsqu'il retourne en Jamaïque pour les vacances (Stolzoff, 2000). Puis il ouvre un dancehall dans «sa " circonscription de Kingston-ouest, plus tard un important studio d'enregistrement (West Indies Recording Limited, WIRL) (Cruse, 2009). Durant son règne en tant que premier ministre, il enverra «ses » artistes représenter la Jamaïque sur les podiums internationaux, notamment son ingénieur Byron Lee, dont le répertoire se situe largement sous la ceinture (Lee, 2003).

Bien que beaucoup ait changé - ce qui n'a rien de surprenant en près de 4 siècles -, le dancehall conserve sa base fondamentale : son public recherche un mélange de grivoiseries, de chansons d'amour et de résistance au système de domination économique. Le "rasoir en marche» Peter Tosh ajoutera à cette trilogie la lutte pour la légalisation de la ganja à partir de 1976. Parmi le flot d'obscénités déversé par les artistes 
à la mode, nul ne peut échapper au message de résistance qui perdure, principalement la remise en question de l'hégémonie politique de l'élite économique jamaïcaine. En Jamaïque, les descendants des colons britanniques se sont assurés du pouvoir en fondant les deux syndicats, puis les deux seuls partis délimitant le choix «démocratique», et enfin en achetant les votes par la distribution de logements - légalisations de squats ou programmes de rénovations urbains - d'armes et de petits emplois (Cruse, 2009).

La géographie politique de Kingston reflète parfaitement cette caractéristique (Eyre, 1984 ; Figueroa, Sives, 2003). Dans les quartiers de Tivoli ou de Denham Town, on vote par exemple à plus de $99 \%$ (avec des pointes à plus de 105\% les bonnes années !) pour le parti conservateur (Jamaican Labor Party - JLP). À un jet de pierre, dans les quartiers de Jones Town et de Arnett Gardens, 95 à 100\% des électeurs votent pour l'ancien parti socialiste (People national Party - PNP) (Cruse, 2009). Tous les bidonvilles de la capitale se caractérisent par ce découpage politique particulièrement sanglant dans les zones tampons comme Trenchtown. C'est dans ce quartier, sur la «ligne de front» entre les gangs des deux partis qu'est né le reggae dans les arrière-cours de la première rue où se réunissaient régulièrement Bob Marley, Joe Higgs, Bunny Wailers, Toots, Alton Elis, et de nombreuses autres stars de la musique jamaïcaine. Le reggae jamaïcain est ainsi né dans un véritable "étau géopolitique ", resserré par la tectonique des " garnisons ", un espace dans lequel la "guerre froide" s'est matérialisée par des centaines de meurtres commis au pic à glace, avec des revolvers artisanaux, ou avec les armes automatiques dont la CIA inonda le pays dans l'espoir d'endiguer le mouvement socialiste poussé par Michael Manley. La lutte entre le reggae conscient des années 1970, soutenu par le parti socialiste (qui le soutiendra timidement en retour), et le dancehall slack (notamment) des années 1980, soutenu par le parti conservateur d'Edward. Seaga, représente la projection de cet affrontement idéologique dans la sphère culturelle. Le "mur de Berlin », surnom utilisé en Jamaïque pour désigner la "ligne de front", les sépare (Cruse, 2009). Le symbole le plus fort de cet affrontement demeure le discours prononcé par E. Seaga, fraîchement élu premier ministre, lors de la cérémonie funéraire en l'honneur de Bob Marley. Le nouveau Premier Ministre enterre littéralement le reggae conscient...

\section{La généralisation de la culture musicale du clash révélatrice de l'évolution de la société jamaïcaine}


Photographie 1 - Inscription murale délimitant l'aire d'influence du gang Spanglers lié au People National Party (PNP), Crossroad, Kingston, 2009

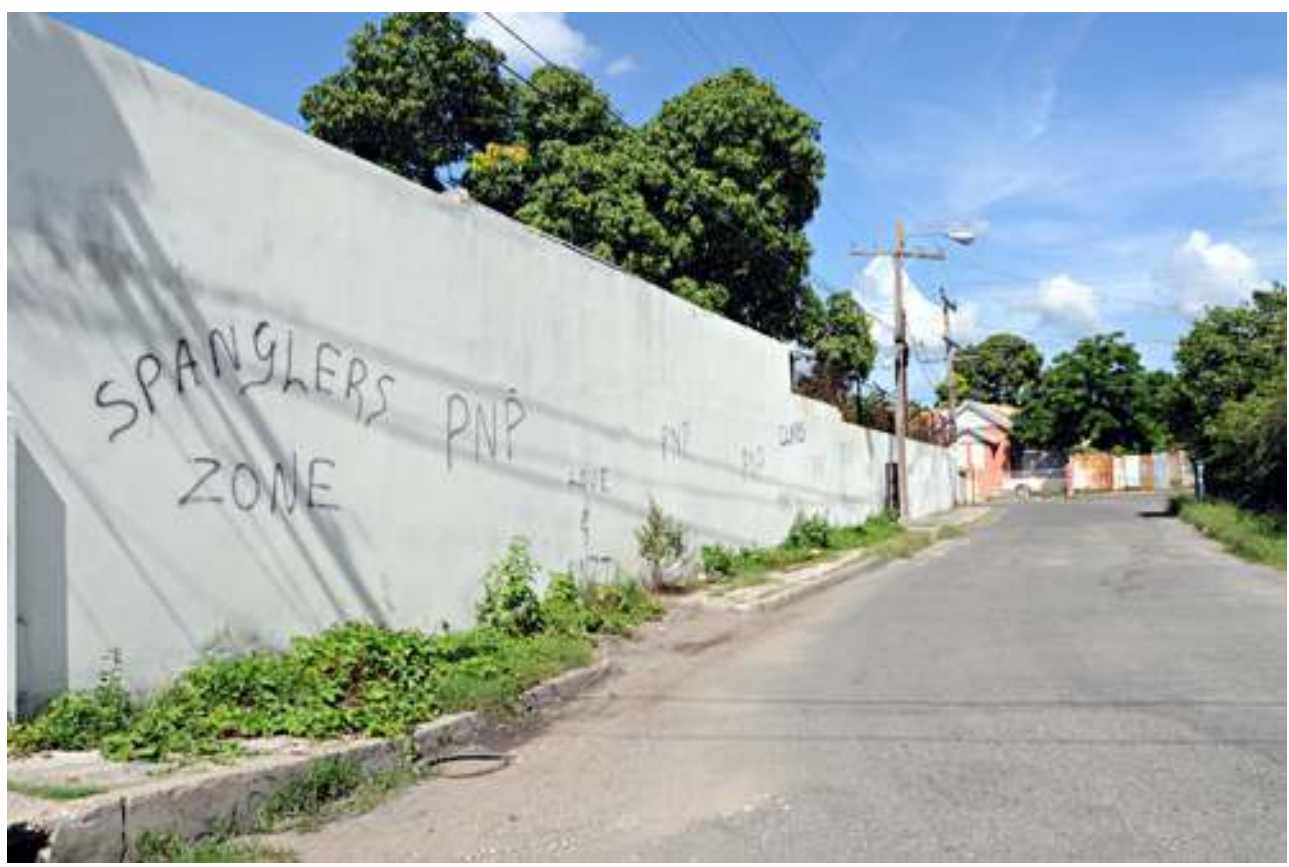

Source : Romain Philippon

Aujourd'hui la capitale toute entière est divisée en zones de guerres régies par les gangs politiques, des gangs de trafiquants de drogues illicites et des gangs de déportés, expulsés des États Unis après un séjour en prison fédérale. Ces gangs s'affrontent entre eux pour le contrôle des rares secteurs lucratifs que sont principalement le trafic de ganja et de migrants clandestins, ainsi que le racket des commerces (Figueira, 2004). De manière relativement semblable à la situation de villes comme Los Angeles, l'espace de ces gangs est délimité par des inscriptions murales (des «tags») au nom du parti ou du gang régnant sur les lieux (Cf. photographie 1).

Cette fragmentation criminelle et politique est doublée d'une fragmentation identitaire musicale - sur les mûrs et dans les esprits -, le ralliement à l'un des chanteurs dominant de la scène du dancehall.

Comme on l'a noté auparavant pour les années 1970/1980, l'espace sonore affiche partiellement la projection de l'espace physique. La culture du clash s'est par exemple développée de manière nouvelle dans les années 1980 - à la suite de la campagne de déstabilisation menée de 1976 à 1980 - autour du nouveau style musical « dancehall » qui, au contraire de la forme musicale précédente (le reggae et ses valeurs d'unité et de résistance), prône l'individualisme, la compétition effrénée et la loi de la jungle. Ceci ne représente pas un bouleversement radical, la violence politique du diviser pour mieux régner débute avant l'indépendance du pays, au début des années 1960, et non pas dans les années 1980, et le reggae connaît son lot de «slack » et d'appels à la violence, mais un changement de tendance, l'évolution d'une moyenne et non l'ensemble des cas.

Progressivement Vivian Blake, leader du célébrissime Shower Posse (célèbre gang politique de la garnison JLP de Tivoli Gardens), introduit les concours de «Dancehall Queen ", à mi chemin entre concours de beauté et compétition de danses suggestives (Blake, 2002). Puis toute la culture du Dancehall se reconvertit progressivement en un art 
du clash, l'opposition sur scène entre artistes, entre sound systems, entre danseuses, etc. Une seule chose compte désormais dans ces pistes de danse reconverties en " arènes »: « tuer» (kill) son adversaire. La guerre déclarée est bien évidemment symbolique mais les danseuses en compétition se jettent rapidement de l'acide au visage, des danseurs célèbres sont assassinés (le célèbre Boggle pour le plus récent), de même en est-il des gérants de sound systems, etc.

Comme les artistes, leurs fans s'opposent - le plus souvent verbalement -, et ils délimitent leur quartier de tags symbolisant leur appartenance (Cf. photographie 2). C'est ainsi qu'à l'heure actuelle (2009) de nombreuses entrées de ruelles sont taguées «Gaza Strip », ce qui ne marque aucune orientation dans le conflit israélo-palestinien mais le rattachement $\mathrm{au}$ " crew" du chanteur Vybz Kartel, originaire du ghetto de "Gaza", à Portmore (banlieue de Kingston). En d'autres lieux des messages menaçants interdisent l'accès à ces "gazamen». Ces zones sont taguées "Gully», diminutif du nom du quartier du second artiste le plus en vogue du moment, l'autoproclamé «Badman for life » et "Gully God » (Littéralement le dieu des égouts) Mavado. Comme le montre l'inscription murale sur l'illustration prise devant la maison familiale de cet artiste (Cf. photographie 3), la superposition entre affrontements identitaires et affrontements politiques peut dans certains cas être frappante.

Ces deux artistes s'affrontent régulièrement sur scène dans des tenues militaires, en prêchant aux adolescents désœuvrés (et armés) un sens de l'honneur qui se défend arme au poing. L'espace du dancehall demeure le miroir de la société jamaïcaine, que l'ONG Nation Master - s'appuyant sur les chiffres proposés par les Nations Unies - classe troisième pays le plus dangereux au monde si on se réfère au ratio de meurtres par habitant, second si on parle du ratio de meurtres commis par des adolescents.

Photographie 2- Une des nombreuses zones Gaza, Trenchtown, Novembre 2009

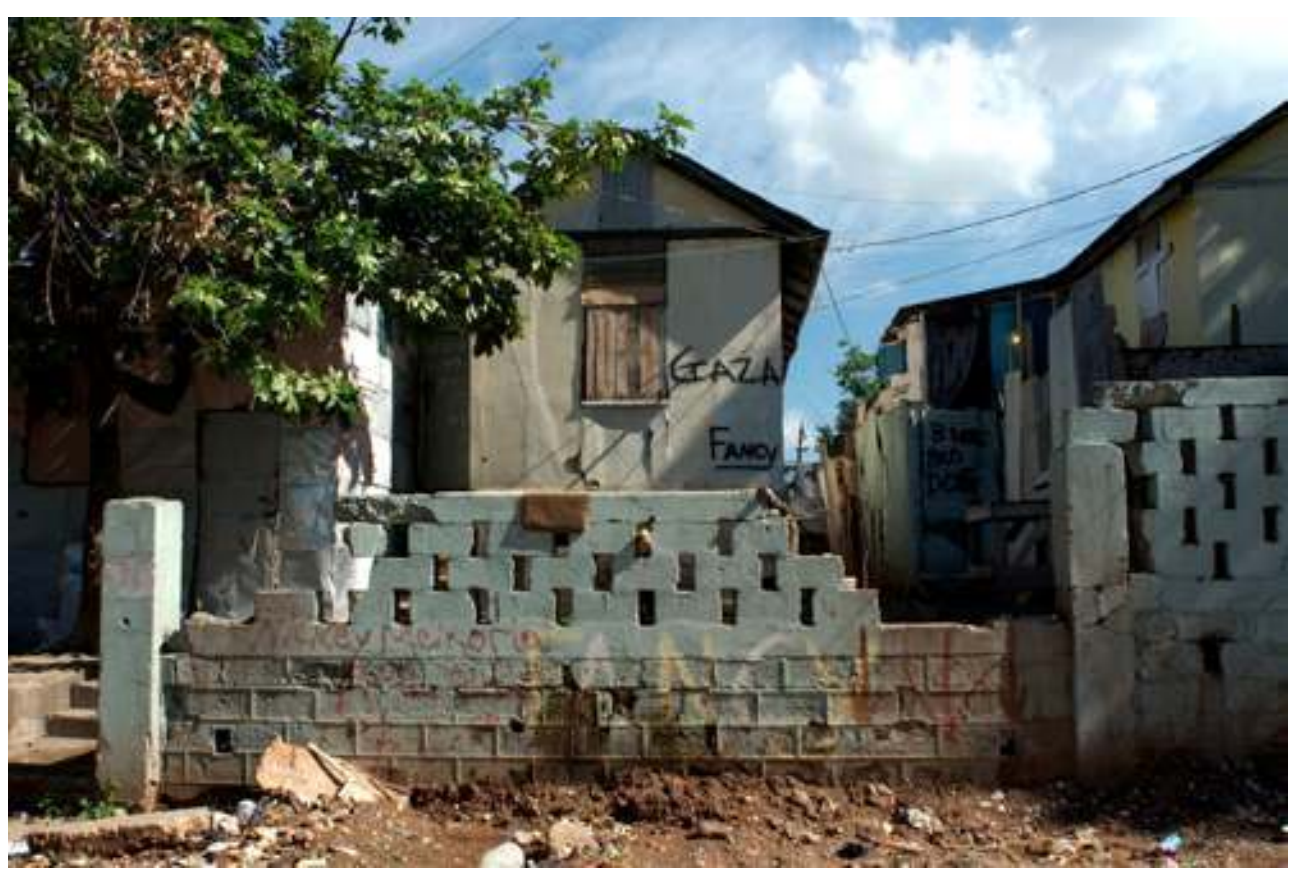

Source : Romain Philippon 


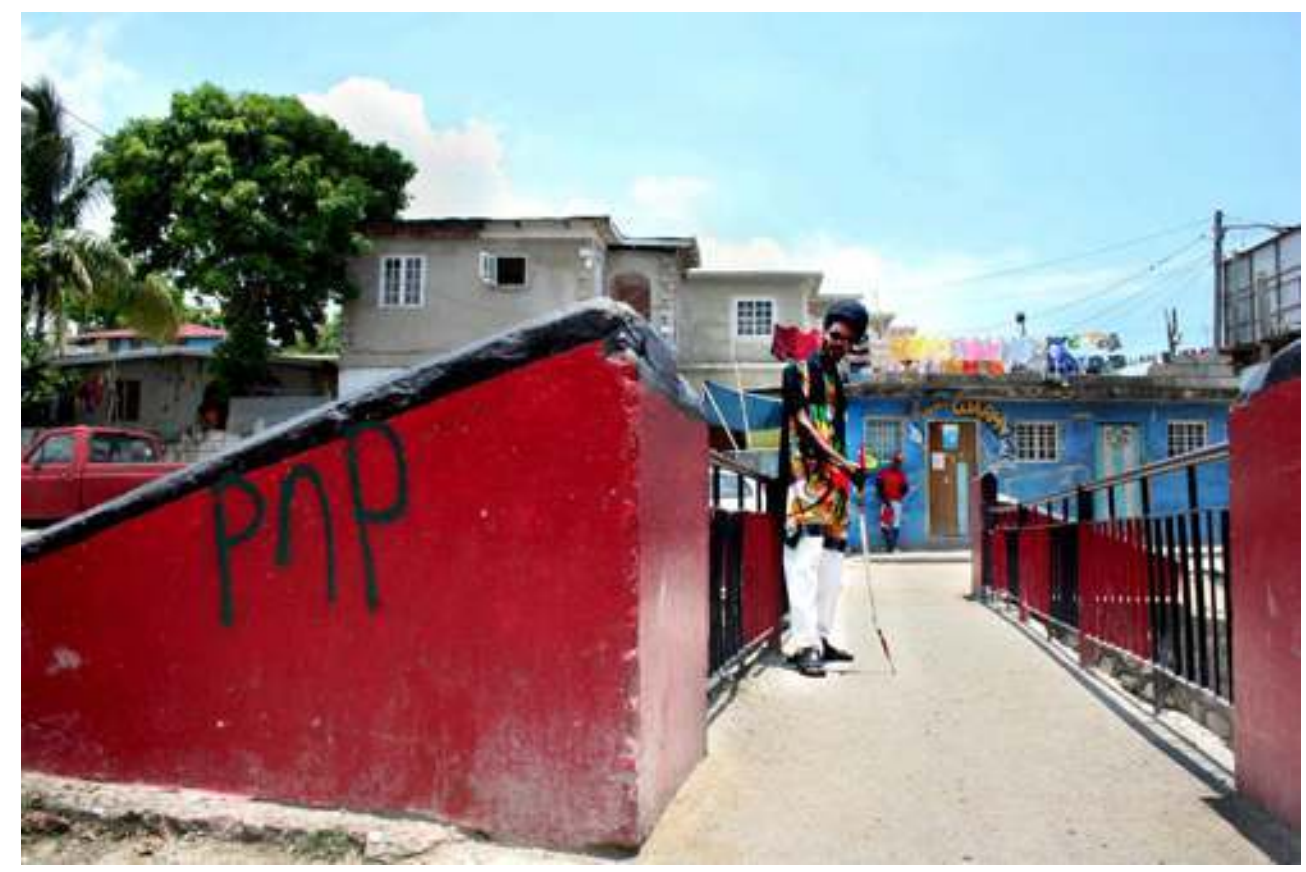

Source : Romain Cruse

L'excitation est à son comble lors du gigantesque concert annuel Sting, le 26 décembre 2008, lorsque Mavado monte sur scène en tenu de «Squadies» (police spéciale) pour affronter lyricalement un Vybz Kartel affublé d'un uniforme militaire complet, et paré d'un cercueil en carton sur lequel est inscrit le nom de son "adversaire». Tout ceci prêterait à sourire si la tension liée à cet affrontement identitaire ne montait pas progressivement dans les quartiers de la ville basse. Les premières victimes de cette "guerre » furent des écoliers, poignardés par des camarades (les écoliers jamaïcains, dès leur plus jeune âge, s'arment de couteaux ou cachent un coutelas dit « cubain » dans leur pantalon - dans le meilleur des cas - pour aller à l'école) à la suite d'une dispute sur leur « appartenance ». Puis la police et les journaux signalèrent des cas de passants agressés dans leur voiture pour avoir joué la mauvaise musique au mauvais endroit. Dans certains quartiers, des groupes de jeunes hommes pénètrent dans des logements privés pour éteindre la musique "ennemie », et font fuir des "selecta " ayant osé bravé l'interdit avant de détruire (ou de voler) leur matériel. Des gérants de Sound System se plaignent d'abus et de violences... (McLean, LeVaughn, 2009 ; Boyne, 2009).

Il est intéressant, pour le chercheur en sciences sociales, de s'intéresser à cette nouvelle fragmentation de l'espace jamaïcain engendrée - ou reflétée, là réside le point central du débat qui anime le pays - par la culture du dancehall. Il semble à première vue que les enfants ayant grandi au cœur des affrontements politiques armés entre garnisons - on se rappelle que de nombreux règlements de compte sous forme de fusillades se produisirent dans les dancehalls à partir du début des années 1980 (Gunst, 1995 ; Blake, 2002) - aient reproduit ce modèle basé sur l'opposition entre quartiers, entre territoires distincts, en dehors de toute lutte idéologique, criminelle ou encore moins d'appartenance ethnique (réelle ou perçue). On se retrouve dans un cas similaire aux sociétés de noirs marrons jamaïcains qui, pour certaines, reproduiront dans leurs territoires des montagnes bleues la «plantalogique» des castes et de la servilité de la société esclavagiste. La forme de 
violence nouvelle associée à un strict attachement identitaire lié au dancehall apporte un argument de plus aux chercheurs décrivant la Jamaïque comme caractérisée par « une culture de la violence immature ", par opposition à l'Italie ou la Chine par exemple. (Harriot, 2003). De l'autre coté de l'échelle sociale, comme on le voit à la lecture des articles récents parus sur le sujet, le "tribalisme politique " a été reconverti par les "uptowners » en " tribalisme musical ", ce qui jette le discrédit une fois de plus sur les populations pauvres et noires. Comme nous l'avons montré ailleurs (Cruse, 2009), le prétendu «tribalisme » associé par la bourgeoisie "claire» jamaïcaine aux classes pauvres afro-jamaicaines renvoie plus à de fallacieux arguments racistes de génétique (sans aucune trace de fondement scientifique) qu'à la réalité sociale d'une société ségrégée. Des chercheurs comme l'anthropologue H. Gayle ont pourtant souligné avec justesse l'origine sociale de ces phénomènes (cité dans McLean, LeVaughn, 2009).

\section{Conclusion}

Le dancehall jamaïcain, que l'on parle du style musical au sens strict ou au sens large (l'espace sonore), ou encore de l'espace physique, le lieu ou se tient l'évènement, fait l'objet de tentatives de territorialisations concurrentes, c'est à dire de rivalités pour la «prise de l'espace ». Il est donc un objet éminemment géopolitique si l'on entend ce terme dans une optique moderne - française - (emboîtement des échelles du phénomène géopolitique jusqu'aux échelles les plus grandes) telle qu'elle est envisagée, dans différents champs d'études, par les spécialistes Y. Lacoste, S. Rosière, ou encore G. Dussouy (Dussouy, 2006 ; Rosière, 2008 ; Lacoste, 2003). Son analyse nous rappelle que la musique caribéenne fait de manière plus générale l'objet d'enjeux (géo)politiques, les liens entre ces deux mondes étant scellés par exemple par le «koudiay» haïtien, la politique culturelle initiée par Jean-Claude «Baby Doc » Duvalier. On détourne l'attention des masses des problèmes politiques cruciaux, eux aussi véhiculés par une musique basée sur le commentaire social, en encourageant et en sponsorisant des groupes, et des styles conservateurs (le célèbre Kompa par exemple, au détriment de la Mizik Rasin de groupes engagés comme Boukman Experiens) (Gage, 2006). On se rappelle, en dehors d'Haïti, de l'attaque par le gouvernement Panday, à Trinidad, des chanteurs les plus engagés du Calypso local (Lashley, 2004 ; Cruse, 2009). De même en Jamaïque où les partis politiques ont largement instrumentalisé les vagues musicales. Le célèbre Bob Marley ira jusqu'à chanter en ouverture de meetings du parti socialiste (PNP) tandis que le leader conservateur E. Seaga envoyait, a chaque fois qu'il en eut l'occasion, «ses" artistes (notamment l'ingénieur de son studio WIRL) promouvoir une vision radicalement opposée du pays sur les scènes internationales (Lee, 1999). Un gang politique issu de "sa » garnison de Tivoli Gardens (où le stade de football porte son nom et les murs son portrait) tentera par ailleurs d'assassiner la plus grande flamme du reggae à la fin des années 1970. Averill Gage et Norman Stolzoff font chacun de leur coté allusion à des situations similaires en République Dominicaine et nulle doute qu'une étude plus détaillée de «musicologie politique » dans la région révèlerait des similarités ailleurs dans les Petites Antilles, à Cuba, en Colombie, au Venezuela, etc.

On le voit dans le cas jamaïcain, les dérives de la culture du clash, encouragée dans les années 1980 en réponse au "reggae conscient " de la décennie précédente et à son message socialisant, débouchent sur une nouvelle forme de justification du recours à la violence en raison d'un étonnant attachement identitaire à des artistes en vogue, 
représentés par la toponymie de leurs quartiers d'origine (qu'ils ont par ailleurs quitté depuis longtemps pour le luxe des beaux quartiers).

Ceci illustre la multidimensionalité d'espaces - plus exactement, donc, de territoires - tels que les dancehalls jamaïcains et leur importance pour l'analyse de ces sociétés dont la complexité est masquée par la brièveté de leur période historique. Ceci illustre en outre la nécessité de "renverser» le point de vue du géographe, à travers des démarches clairement et ouvertement "post-coloniales", et de commencer à renverser la perspective de l'analyse, ou étudier les sociétés "par le bas", pour reprendre une mauvaise métaphore sociale.

\section{BIBLIOGRAPHIE}

Adams, E., 1991. Understanding jamaican patois, an introduction to afro-jamaican grammar, LMH publishing, $109 \mathrm{p}$.

Averill G., 1997. A day for the hunter, a day for the prey, Popular music and power in Haiti, University of Chicago Press, $306 \mathrm{p}$.

Barrow S., Dalton P., 1997. Reggae, the rough guide, London, Rough guide.

Blake D., 2002. Shower Posse, the most notorious jamican criminal organisation, New York, Diamon Publishing, $403 \mathrm{p}$.

Boyne I., 2009. The gully-gaza war, Jamaica Gleaner, 20 septembre 2009.

Cassidy F., 1971. Jamaica talk : three hundred years of english language in Jamaica, London, MacMillan Caribbean.

Cooper C., 1994. "Lyrical Gun": Metaphor and Role Play in Jamaican Dancehall Culture, The Massachusettes Review, Vol 37, N³, p. 429-447

Cooper C., 2000, Noises in the blood: Orality, gender and the 'vulgar' bo dy of Jamaican popular culture, Durham, Duke University Press.

Cruse R., 2009. L'antimonde caribéen, entre les Amériques et le monde, Thèse de doctorat, Université d'Artois, $730 \mathrm{p}$.

Cruse R., 2008. Médailles d'or jamaïcaines, Le Monde Diplomatique, Août 2008.

Dussouy G., 2006. Les théories géopolitiques, traité de relations internationales (1), L'Harmattan, $363 \mathrm{p}$.

Eyre A., 1984. Political violence and urban geography in Kingston, Jamaica, American Geographical Society, http://www.jstor.org/pss/214758

Farmer P., 2006. The uses of Haiti, Monroe, Comon Courage Press, 478 p.

Figueira D., 2004. Cocaine and Heroin Trafficking in the Caribbean, Vol I, Trinidad, Guyana, Jamaica, New York, Iuniverse Inc., 213p. 
Figueroa M., Sives A., 2003. « Garrison politics and criminality in Jamaica, does the 1997 election represent a turning point ? ", in Harriott, A., (Ed.), Understanding crime in Jamaica, new challenges for public policies, Kingston, UWI Press, p. 63-89.

Gunst L., 1995. Born fi dead, A journey through the jamaican posse underworld, Owl Book, New York, $246 \mathrm{p}$.

Harriot A., 2003. "Social Identities and the escalation of homicidal Violence in Jamaica", in Harriot, A., (ed) Understandig crime in Jamaica, new challenge for public policies, Kington Jamaica, UWI Press, p. 89-112.

Harris W., 1970. History, Fable and Myth in the Caribbean and Guianas, New York, Calaloux publications, $50 \mathrm{p}$.

Katz D., 2003. Solid foundation, an horal history of reggae music, New York, Blumsbury.

Lacoste Y., 2003. De la géopoltique aux paysages, dictionnaire de la Géographie, Paris, Armand Collin, $413 \mathrm{p}$.

Lashley L., 2004. Intimidation of Calypsonians by the UNC government of trinidad and Tobago, Proudflesh, www.proudfleshjournal.com/issue3/lashley.htm

Lee H., 1999. Le premier rasta, Etonnants voyageurs, Flammarion, $367 \mathrm{p}$.

Lee H., 2004. Voir Trenchtown et mourir, Flammarion, 395 p.

Lesser B., 2008. Dancehall, The story of Jamaican Dancehall culture, Soul jazz records publishing.

McLean R., LeVaughn F., 2009. Seeing beyond the Gaza/Gully smoke screen: The social issues underlying dancehall tribalism, Jamaica Gleaner, 13 septembre 2009.

Mullings B., 1999. « Globalization, Tourism and the International Sex Trade », in Kempado K., Sun, sex, and gold. Tourism and sex work in the Caribbean, New York, Oxford, Boulder, Rowman \& Littlfield Publishers, p. 55-80.

O'Connell J., Taylor J., 1999. « Fantasy Island, exploring the demand for sex tourism », in Kempado K., Sun, sex, and gold. Tourism and sex work in the Caribbean, New York, Oxford, Boulder, Rowman \& Littlfield Publishers, p. 37-54.

Rosière S., 2008. Dictionnaire de l'espace politique, Géographie politique et Géopolitique, Armand Colin, $319 \mathrm{p}$.

Stolzoff N., 2000. Wake the town and tell the people, dancehall culture in Jamaica, Durham, Londres, Duke University Press, 290 p.

\section{NOTES}

1. Comme nous le verrons par la suite, les frontières du dancehall correspondent étroitement avec une frontière, ou plus exactement une transition linguistique (isoglosse). L'anglais britannique y cède progressivement la place au créole afrojamaïcain..

2. Le terme « créole » est lui même polysémique et employé dans des sens très différents dans la Caraïbe. Ainsi, un créole est à l'origine un planteur blanc « des îles». Rapidement le terme laisse cependant entendre une forme de métissage. A tel point que le terme "créole" désigne aujourd'hui en Guyane Française ou encore dans l'île anglophone de Trinidad (par exemple) les populations afro-caribéennes plus ou moins métissées (à différencier des «noirs marrons » dans le cas de la Guyane, mais aussi du Suriname voisin ou du Guyana, et des indo-caribéens à Trinidad et au Guyana). Dans la littérature et dans la poésie Antillaise francophone (qu'on parle de Aimé 
Césaire, de Raphael Confiant de Gary Victor ou de René Depestre pour ne citer que quelques auteurs), le terme de "créolisation » a ensuite été utilisé comme synonyme de métissage et c'est dans ce sens que nous l'employons dans cet article.

3. Un béké est un créole dans le sens originel, c'est à dire un descendant de planteur blanc, non métissé.

4. Interview vidéo disponible sur : http://www.gwadeloupe.com/blog/video-bekes--les-derniersmaitres-de-la-martinique

\section{RÉSUMÉS}

Cet article examine la polysémie du dancehall jamaïcain entendu à la fois comme musique - ou espace sonore - et, au sens littéral, comme lieu particulier sur lequel se tiennent les « sessions ». On s'aperçoit à travers l'analyse que le dancehall, dans toutes ses acceptations, est un espace convoité, disputé et territorialisé car objet d'enjeux identitaires, économiques (formels et informels) et politiques. La prise de l'espace étant l'objet principal, on s'intéressera aussi à une géopolitique de grande échelle des dancehalls jamaïcains.

This paper looks at the polysemy and scales of jamaican dancehall as musical style(s) as well as a place that holds the 'sessions'. The study shows that dancehall is quite a disputed place mainly due to its influence in the realm of identities, economics (both formal and informal) and politics. 'Holding the place' being such a central issue, we will as well look at large scale geopolitics of jamaican dancehall(s).

\section{INDEX}

Mots-clés : musique, dancehall, Jamaïque, identité, (géo)politique

Keywords : music, Jamaica, identities, (geo)politics

\section{AUTEUR}

\section{ROMAIN CRUSE}

Romain Cruse est Docteur en Géographie - Université d'Artois, Chargé de cours/chargé de recherches - University of the West Indies, Kingston, Jamaica, Chargé de cours - Université des Antilles-Guyane, Fort-de-France.kermarron@gmail.com 